\title{
Comparison of the neurobiological effects of attribution retraining group therapy with those of selective serotonin reuptake inhibitors
}

\author{
C. Wang ${ }^{1,2}$, N. Zhang ${ }^{1}$, Y.L. Zhang ${ }^{2}$, J. Zhang ${ }^{1,2}$, H. Yang ${ }^{1}$ and T.C. Timothy ${ }^{3}$ \\ ${ }^{1}$ Medical Psychology Department, Nanjing Brain Hospital, Nanjing Medical University, Nanjing, China \\ ${ }^{2}$ Mental Health Institute, Second Xiangya Hospital, Central South University, Changsha, China \\ ${ }^{3}$ Massachusetts General Hospital, Boston, MA, USA
}

\begin{abstract}
The aim of this study was to compare the effectiveness of attribution retraining group therapy (ARGT) with selective serotonin reuptake inhibitors (SSRIs) in the treatment of major depressive disorder (MDD), generalized anxiety disorder (GAD), and obsessive-compulsive disorder (OCD). Subjects were sequentially recruited and randomized into two groups, one receiving ARGT $(n=63)$ and the other SSRIs $(n=66)$ for 8 weeks. Fifty-four ARGT outpatients with MDD $(n=19)$, GAD ( $n=19)$, and $\operatorname{OCD}(n=16)$ and 55 SSRI outpatients with MDD $(n=19), \operatorname{GAD}(n=19)$, and OCD $(n=17)$ completed the study. All subjects were assessed using the Hamilton Depression Scale and Hamilton Anxiety Scale before and after treatment. The 10item Yale-Brown Obsessive Compulsive Scale was employed only for OCD subjects. Plasma levels of serotonin, norepinephrine, cortisol, and adrenocorticotropic hormone were also measured at baseline and 8 weeks after completion of treatment. Symptom scores were significantly reduced $(P<0.001)$ in both the ARGT and SSRI groups at the end of treatment. However, MDD, GAD and OCD patients in the ARGT group had significantly lower plasma cortisol concentrations compared to baseline $(P<0.05)$, whereas MDD and OCD patients receiving SSRIs showed significantly increased plasma levels of serotonin $(P<0.05)$. These findings suggest that ARGT may modulate plasma cortisol levels and affect the hypothalamuspituitary-adrenal axis as opposed to SSRIs, which may up-regulate plasma serotonin levels via a different pathway to produce an overall improvement in the clinical condition of the patients.
\end{abstract}

Key words: Attribution retraining group therapy; Selective serotonin reuptake inhibitors; Neurobiological effects; Serotonin; Cortisol

\section{Introduction}

Currently the two main treatments in clinical practice for major depression disorder (MDD), generalized anxiety disorder (GAD) and obsessive-compulsive disorder (OCD) are antidepressants and psychotherapy. Many studies examining the use of antidepressants in the treatment of psychiatric disorders focus on the underlying neurobiological mechanisms, whereas most studies on psychotherapy focus on its effects on symptom management and psychosocial function. Few studies have examined the role of psychotherapy in improving neurobiological function (1-3), with even fewer studies comparing the difference between the two therapies in terms of their effects on neurobiological function.

Over the past few decades a number of studies have demonstrated the crucial role of neurotransmitters and neuroendocrine function in the psychopathology of MDD,
GAD, and OCD. The central hypothesis for the pathophysiology of these depressive disorders is primarily related to serotonin (5-hydroxytryptamine, 5-HT) and norepinephrine (NE). Abnormal 5-HT and NE levels have been reported in the three diseases. Most studies have indicated lower 5-HT levels for MDD patients and higher NE levels for GAD patients than for normal subjects (4-6). The neuroendocrine dysfunction hypothesis mainly refers to abnormal hypothalamus-pituitary-adrenal (HPA) axis function, such as abnormal activities of adrenocorticotropic hormone (ACTH) and cortisol (7-10).

Several studies imply that successful psychotherapy may have a positive effect on neurotransmitters, including 5-HT, NE and their metabolites, for normal subjects and MDD patients $(2,3,11,12)$. However, the outcomes of these studies are varied and some studies have been

Correspondence: N. Zhang, Nanjing Brain Hospital, Nanjing Medical University, Nanjing 210029, China. Fax: +86-25-83719926. E-mail: zn6360@126.com and Y.L. Zhang, Mental Health Institute, Second Xiangya Hospital, Central South University, Changsha 410011, China. Fax: +86-731-5292159. E-mail: zhangyl69@vip.sina.com 
conducted on small patient samples. Data from some studies support the hypothesis that plasma 5 -HT levels increase after psychotherapy (3) while others have not shown a significant change in plasma 5-HT levels (11). One study reported that NE metabolite levels decrease after psychotherapy (11). Another study demonstrated a nonsignificant decrease in plasma NE metabolite levels during treatment in cognitive therapy responders compared with non-responders (12) while other studies have suggested that psychotherapy may affect blood levels of ACTH and cortisol $(1,13,14)$. Abelson et al. (14) found that cognitive/emotional manipulation can substantially modulate the responses of the HPA axis to pharmacological activation in normal subjects and in patients with panic disorder via a pharmacological activation paradigm (14).

Attribution retraining (AR) is one of a number of therapeutic approaches classified as cognitive behavior therapy. AR is designed to change maladaptive attribution styles to more adaptive ones (15). Individuals attribute behaviors and events to various reasons. Hence the attributional style of an individual is defined as that individual's characteristic way of explaining the cause of events. The basis of AR therapy centers around the modification of this thought process with the ultimate aim of modifying behavior $(15,16)$. AR is based on the integrated hopelessness/self-esteem theory proposed by the groups of Abramson, Seligman, and Metalsky (17-19). In AR interventions, therapists usually target the patient's automatic thoughts, which are rooted in unhealthy attributions, and challenge these pessimistic attributions by offering alternative explanations based on healthy attributions (15). Hence AR can be applied to a variety of psychological problems with maladaptive attributional style (15). A number of studies have demonstrated a link between maladaptive attributional style and various psychological problems, including depression and anxiety (20-23).

In China, Wang and Zhang $(24,25)$ developed a group form of AR named AR group therapy (ARGT), which is based on the integrated hopelessness/self-esteem theory and AR. ARGT is a form of group cognitive-behavior therapy that treats clients' maladjusted emotions and behaviors by changing their rationalization strategy explanations for problems and symptoms. They examined ARGT in Chinese college graduates with depression and outpatients with MDD, GAD, and OCD and successfully demonstrated that ARGT was able to reduce their symptoms and alter their maladaptive attributional styles (25-27).

The purpose of the present study was 1) to characterize the neurobiological effects of ARGT on plasma levels of $5-\mathrm{HT}, \mathrm{NE}, \mathrm{ACTH}$, and cortisol for outpatients with MDD, $G A D$, and $O C D$, and 2) to compare the neurobiological effects obtained with ARGT to those obtained with 5serotonin reuptake inhibitors (SSRI) antidepressants.

\section{Material and Methods}

The study was approved by the Ethics Committee of Nanjing Brain Hospital, Nanjing Medical University (China) before patient recruitment, and written informed consent was obtained from all participants at the time of recruitment.

\section{Participants}

Subjects aged 16 50 years who met the DSM-IV criteria for MDD, GAD or OCD based on the Structured Clinical Interview for DSM-IV Axis I disorders, patient edition (SCID-I/P, Version 2.0, China) (28) were recruited among outpatients presenting to a mental health hospital in Nanjing, China, between October 2007 and September 2008. The diagnostic interview was held by two attending psychiatrists with more than 6 years of clinical experience.

The inclusion criteria for the study were: 1) MDD group: scores $\geqslant 18$ on the 24-item version of the Hamilton Rating Scale for Depression (HAMD); 2) GAD group: scores $\geqslant 14$ on the 14-item version of the Hamilton Rating Scale for Anxiety (HAMA); 3) OCD group: scores $\geqslant 16$ on the Yale-Brown Obsessive-Compulsive Scale (Y-BOCS); 4) willing to participate in the study for 8 weeks.

The exclusion criteria were: 1) neurological disease, 2) serious physical illness (e.g., heart, lung, liver, kidney, or blood system disease), 3) drug or alcohol abuse, 4) psychotic symptoms, 5) personality disorders, 6) pregnancy, 7) suicide risk, 8) treatment with antidepressants or other psychotropic medications within 6 months prior to the beginning of the trial; 9) more than a single target diagnosis (e.g., comorbidity for MDD and GAD or MDD and OCD).

The criteria for exclusion during the investigation were: 1) failure to attend psychotherapy or non-adherence to antidepressant medication for 2 consecutive weeks, 2) serious adverse drug reaction events or requirement of antidepressant withdrawal because of adverse drug reactions, 3) need for other treatment outside the study because of attempted suicide, poor feeding, etc., 4) serious physical illness or infectious diseases during the course of the study, 5) pregnancy, 6) significant changes in life, 7) incorrect diagnosis, 8) withdrawal of informed consent.

\section{Study design}

A clinical trial study design was used. Outpatients with MDD, GAD, and OCD were sequentially allocated to the ARGT group or SSRI treatment group by a block randomization with a block size of 8 (since there are 8 patients in each ARGT subgroup). Response to treatment was assessed with symptomatology scales in both groups. Blood samples were also collected before and after intervention to measure plasma levels of $5-\mathrm{HT}, \mathrm{NE}$, cortisol, and ACTH. 


\section{Assessments}

Measurements were made at the time of recruitment (baseline; week 0) and at the final week of the treatment course (post-test; week 8). The 24-item version of HAMD (29) was used to measure the severity of depressive symptoms in MDD subjects. The internal consistency of the Chinese version of HAMD is $0.88 \sim 0.99$ and the authenticity coefficient (reflecting the severity of clinical symptoms) is 0.92 . The 14-item HAMA (30) was used to measure the severity of anxiety symptoms for GAD subjects. The inter-rater reliability of the Chinese version of HAMA is 0.93 and the authenticity coefficient is 0.92 . The 10-item Y-BOCS (31) was employed to assess the severity of obsessions and compulsions in OCD subjects. The inter-rater reliability (intraclass correlation coefficient) is $\geqslant 0.82$, the test-retest reliability (intraclass correlation coefficient) is $\geqslant 0.82$ and the Cronbach alpha reliability is 0.75 . The scale has good content validity and construct validity.

Assessment was blind. All the informant-report questionnaires were administered by two psychologists who did not know the aim of this study. All staff administering the assessments received specific professional training for each assessment for more than one month prior to the beginning of the study. The Spearman correlation coefficients (HAMD, HAMA, Y-BOCS) between the two psychologists were $0.832,0.835$, and 0.931 .

Demographic data (age, gender, marital status, educational level, family environment) and clinical characteristics (onset of the disease, stressful life events, course of the disease, psychotropic medication history, psychotherapy history, family history, and physical illness history) were also collected at the time of recruitment.

\section{Blood samples}

Subjects were required to observe a tyramine-free diet (no alcohol, cheese or coffee) for $48 \mathrm{~h}$ before phlebotomy and to keep a record of all food eaten during this period. After an overnight fast, blood samples were drawn between 8 and 10 o'clock in the morning at the time of recruitment (baseline) and by the 8 th week (post-test). Blood samples $(5 \mathrm{~mL})$ were drawn into tubes containing heparin anticoagulant and centrifuged at $4200 \mathrm{rpm}$ for $4 \mathrm{~min}$ at $4^{\circ} \mathrm{C}$ after a 30 -min interval. The supernatant plasma was extracted and stored frozen at $-80^{\circ} \mathrm{C}$ until the time of assay.

Plasma levels of 5-HT, NE, cortisol, and ACTH were determined by a radioimmunoassay method. Commercially available kits were used to quantify plasma hormone levels. 5-HT and NE kits (Biosource Europe SA, Belgium) had standard ranges of $15 \sim 2500$ and $4.5 \sim 450 \mu \mathrm{g} / \mathrm{L}$, sensitivity of 0.3 and $0.0375 \mu \mathrm{g} / \mathrm{L}$, and a coefficient of variation (CV) $<5$ and $<10 \%$, respectively. The ACTH kit (Diagnostic System Laboratories, USA) had a standard range of $6.0 \sim 407.4 \mathrm{ng} / \mathrm{L}$, sensitivity of $1.3 \mathrm{ng} / \mathrm{L}$ and $\mathrm{CV}<10 \%$. The cortisol kit (Beijing Chemclin Biotech Co., Ltd., China) had a standard range of $10 \sim 500 \mu \mathrm{g} / \mathrm{L}$, sensitivity of $2 \mu \mathrm{g} / \mathrm{L}$ and $\mathrm{CV}<10 \%$. Absolute levels were quantitated with a gamma radioimmunoassay counter (GC-911, Science and Technology Development Co., China) by professional and technical staff at the Neuropsychiatric Institute, Nanjing Brain Hospital affiliated to Nanjing Medical University.

\section{Treatment}

Patients randomized to the ARGT group received ARGT once a week according to a previously validated protocol (24-27). Antidepressant medications were withheld for the duration of the ARGT. Each ARGT session lasted $2 \mathrm{~h}$ and was held weekly over an 8-week course. Participants were allocated to different ARGT subgroups according to the sequence of enrolment in the study, with 7 8 patients allocated to each ARGT subgroup. Within a structured therapy protocol, each session focused on a specific topic as follows: 1) knowing and supporting each other; 2) the meaning of symptoms and the effects of cognitive factors; 3 ) the role of attribution in psychology; 4) participants' upbringing and basic beliefs; 5) rebuilding attributional styles and practicing new behaviors; 6) consolidating new attribution styles and behaviors; 7) self-esteem, personality and attributions for positive events; 8) sharing future plans and discussing leaving.

ARGT was performed by two therapists for each subgroup. One was the main therapist who coordinated all subgroups. The other was the co-therapist. There were different co-therapists for different subgroups. All therapists had completed training in psychotherapy. The main therapist was a clinical and counseling psychologist registered in the Professional Organizations and Individual Practitioners in Clinical and Counseling Psychology, Chinese Psychological Society. The cotherapists were blind to the purpose of the study. Additionally there were 4 supervisors who were registered psychologist supervisors from the Chinese Psychological Society who supervised the treatment sessions. Each ARGT subgroup participated in a session supervised by one of the psychologist supervisors at least once every two weeks. To maintain treatment integrity, the cotherapist also recorded each step of the intervention plan and reported to the main therapist prior to the subsequent session.

Patients in the SSRI group received standard clinical treatment with one of the following SSRI antidepressants: fluoxetine (Prozac), paroxetine (Paxil), sertraline (Zoloft), citalopram (Celexa), and fluvoxamine (Luvox), and did not receive psychotherapy during the study period. The choice of antidepressant depended on the symptoms and tolerance. Medications were titrated from the minimum effective doses upwards. The initial doses were: $10 \mathrm{mg}$ fluoxetine, $10 \mathrm{mg}$ paroxetine, $50 \mathrm{mg}$ sertraline, $10 \mathrm{mg}$ citalopram, and $50 \mathrm{mg}$ fluvoxamine. The therapeutic doses were: $20 \mathrm{mg}$ fluoxetine, $20 \sim 40 \mathrm{mg}$ paroxetine, $50 \sim 150 \mathrm{mg}$ sertraline, $20 \sim 40 \mathrm{mg}$ citalopram, and 
50 150 mg fluvoxamine. Medication was increased from the initial dose to the therapeutic dose within 2 weeks. After treatment, the average maximum doses were: 20.00 $\pm 0.00 \mathrm{mg}$ fluoxetine, $24.67 \pm 6.40 \mathrm{mg}$ paroxetine, 87.5 $\pm 29.46 \mathrm{mg}$ sertraline, $26.36 \pm 6.74 \mathrm{mg}$ citalopram, and $91.07 \pm 23.22 \mathrm{mg}$ fluvoxamine. The antidepressant treatment was monitored by two clinicians experienced in the use of SSRI.

\section{Statistical analysis}

Data analyses were performed using the Statistical Package for the Social Sciences (SPSS) for Windows, version 15.0 (SPSS Inc., USA). Baseline data were compared between groups in relation to demographic variables using the $\chi^{2}$ test for nominal variables and the independent-samples $t$-test for continuous variables (after the Kolmogorov-Smirnov Z-test and the Levene test). All scores of the continuous variables were tested by the Kolmogorov-Smirnov Z-test for normal distribution and by the Levene test for homogeneity of variance. Nonparametric tests were used for non-normal and non-homogeneous variance data. Normal and homogeneous variance data were tested for significance using the $t$-test. The pairedsamples $t$-test was used to compare the scores at baseline and at week 8 in each group. The independent-samples $t$ test was used to compare reduction scores between two groups. All tests were two-tailed, with the level of significance set at 0.05 .

\section{Results}

\section{Baseline data}

A total of 129 eligible outpatients with MDD $(n=45)$, GAD ( $n=45)$, and OCD $(n=39)$ were enrolled in the study. Sixty-three subjects were randomized to the ARGT group and 66 to the SSRI group. Of the 129 enrolled subjects, 54 outpatients in the ARGT group with MDD (n $=19)$, GAD ( $n=19)$, and OCD $(n=16)$ and 55 outpatients in the SSRI group with MDD $(n=19)$, GAD ( $n$ $=19)$, and OCD $(n=17)$ completed the study. Nine outpatients in the ARGT group (14.3\%) and 11 outpatients in the SSRI group (16.7\%) dropped out of the study. The dropout rate did not differ significantly between the two groups $\left(\chi^{2}=0.139, \mathrm{P}=0.709\right)$.

The demographic and clinical characteristics were collected and compared between the ARGT group and SSRI group (see Table 1). The two groups were well matched in all the characteristics examined, including age, gender, marital status, educational level, diagnosis, onset of illness, stressful life events, course of disease, psychotropic medication history, psychotherapy history, family history, and physical illness history $(P>0.05)$.

\section{Changes of symptomatology scale scores from baseline to the end of treatment}

The Kolmogorov-Smirnov Z-test for normal distribution was applied to the HAMD scores of MDD patients, the HAMA scores of GAD patients, and the Y-BOCS scores of OCD patients in each group. All the variables displayed normal distribution $(P>0.05)$. After the Levene test for homogeneity of variance, the paired-samples $t$-test was used to compare the mean scores at baseline and at week 8 in each group. Table 2 shows the mean pre- and post-scores and the paired-samples $t$-test outcomes. The outcomes showed that the depressive, anxious and obsessive-compulsive symptoms improved significantly after both SSRI and ARGT treatments.

\section{Changes of plasma biological index levels at baseline and after treatment}

Plasma levels of $5-\mathrm{HT}$, NE, cortisol, and ACTH were tested by the Kolmogorov-Smirnov Z-test for normal distribution and by the Levene test for homogeneity of variance. To compare the mean levels at baseline and at week 8 in each group, the Wilcoxon test was used for nonnormal and non-homogeneous variance data while the paired-samples $t$-test was used for normal and homogeneous variance data. The outcomes showed that plasma cortisol levels were significantly reduced in MDD, GAD and OCD patients in the ARGT group, while plasma 5 -HT levels were significantly increased in MDD and OCD patients and plasma NE levels were significantly increased in MDD patients in the SSRI group (see Table 3).

\section{Comparison of score reductions of plasma biological index levels between the ARGT and SSRI groups}

The score reductions of plasma 5-HT, NE, cortisol, and ACTH levels in each group were tested by the Kolmogorov-Smirnov Z-test for normal distribution and the Levene test for homogeneity of variance. To compare the mean score reductions between the ARGT group and the SRI group, the Mann-Whitney U-test was used for nonnormal and non-homogeneous variance data and the independent-samples $t$-test was used for normal and homogeneous variance data. The outcomes showed a significant difference in score reductions of plasma cortisol levels in GAD and OCD patients. No significant difference was found for other score reductions (see Table 4).

\section{Discussion}

The present study demonstrates that ARGT and SSRI can both improve depressive symptoms in MDD patients, anxious symptoms in GAD patients, and obsessivecompulsive symptoms in OCD patients, in agreement with previously published studies $(26,27,32)$. Additionally, this study also explored the neurobiological effects of the two therapies and obtained three major results.

The first was that plasma cortisol levels dropped significantly in patients with MDD, GAD, and OCD after ARGT. Plasma ACTH levels also dropped but the 
Table 1. Demographic and clinical characteristics of the study participants.

\begin{tabular}{|c|c|c|c|}
\hline & ARGT group $(n=54)$ & SSRI group $(n=55)$ & Statistics \\
\hline Age & $29.31 \pm 9.78$ & $30.95 \pm 10.18$ & $\begin{array}{r}0.087^{a} \\
-0.853^{b}\end{array}$ \\
\hline \multicolumn{4}{|l|}{ Gender } \\
\hline Male & $24(44.4 \%)$ & $26(47.3 \%)$ & $0.088^{\mathrm{C}}$ \\
\hline Female & $30(55.6 \%)$ & $29(52.7 \%)$ & \\
\hline \multicolumn{4}{|l|}{ Marital status } \\
\hline Married & $23(42.6 \%)$ & $29(52.7 \%)$ & $2.426^{d}$ \\
\hline Never married & $29(53.7 \%)$ & $23(41.8 \%)$ & \\
\hline Divorced & $2(3.7 \%)$ & $2(3.6 \%)$ & \\
\hline Widowed & $0(0 \%)$ & $1(1.8 \%)$ & \\
\hline \multicolumn{4}{|l|}{ Educational level } \\
\hline$<9$ years & $3(5.6 \%)$ & $8(14.5 \%)$ & $4.304^{\mathrm{C}}$ \\
\hline $9-12$ years & $12(22.2 \%)$ & $15(27.3 \%)$ & \\
\hline $12-16$ years & $33(61.1 \%)$ & $24(43.6 \%)$ & \\
\hline$>16$ years & $6(11.1 \%)$ & $8(14.5 \%)$ & \\
\hline \multicolumn{4}{|l|}{ Diagnosis } \\
\hline MDD & $19(35.2 \%)$ & $19(34.5 \%)$ & $0.021^{\mathrm{c}}$ \\
\hline$A D$ & $19(35.2 \%)$ & $19(34.5 \%)$ & \\
\hline OCD & $16(29.6 \%)$ & $17(30.9 \%)$ & \\
\hline \multicolumn{4}{|l|}{ Onset of illness } \\
\hline First & $38(70.4 \%)$ & $30(54.5 \%)$ & $2.908^{\mathrm{C}}$ \\
\hline Recurrence & $16(29.6 \%)$ & $25(45.5 \%)$ & \\
\hline \multicolumn{4}{|c|}{ Stressful life events } \\
\hline Yes & $44(81.5 \%)$ & $43(78.2 \%)$ & $0.184^{\mathrm{C}}$ \\
\hline No & $10(18.52 \%)$ & $12(21.8 \%)$ & \\
\hline \multicolumn{4}{|l|}{ Course of disease } \\
\hline$\leq 1$ year & $13(24.1 \%)$ & $24(43.6 \%)$ & $5.047^{\mathrm{C}}$ \\
\hline $1 \sim 10$ years & $33(61.1 \%)$ & $23(41.8 \%)$ & \\
\hline$\geqslant 10$ years & $8(14.8 \%)$ & $8(14.5 \%)$ & \\
\hline \multicolumn{4}{|c|}{ Psychotropic medication history } \\
\hline Yes & $24(44.4 \%)$ & $27(49.1 \%)$ & $0.236^{\mathrm{c}}$ \\
\hline No & $30(55.6 \%)$ & $28(50.9 \%)$ & \\
\hline \multicolumn{4}{|c|}{ Psychotherapy history } \\
\hline Yes & $12(22.2 \%)$ & $8(14.5 \%)$ & $1.072^{\mathrm{C}}$ \\
\hline No & $42(77.8 \%)$ & $47(85.5 \%)$ & \\
\hline \multicolumn{4}{|l|}{ Family history } \\
\hline Yes & $13(24.1 \%)$ & $10(18.2 \%)$ & $0.074^{\mathrm{C}}$ \\
\hline No & $41(75.9 \%)$ & $45(81.8 \%)$ & \\
\hline \multicolumn{4}{|c|}{ Physical illness history } \\
\hline Yes & $16(29.6 \%)$ & $15(27.3 \%)$ & $0.568^{\mathrm{C}}$ \\
\hline No & $38(70.4 \%)$ & $40(72.7 \%)$ & \\
\hline
\end{tabular}

Age is reported as means $\pm S D$. All other characteristics are reported as number with percent in parentheses. ARGT $=$ attribution retraining group therapy; SSRI = selective serotonin reuptake inhibitors; MDD = major depressive disorder; GAD = generalized anxiety disorder; $\mathrm{OCD}=$ obsessive-compulsive disorder. ${ }^{a}$ Levene test; ${ }^{\mathrm{b}} \mathrm{F}$-test; ${ }^{\mathrm{C}}$ Pearson chi-square test; ${ }^{\mathrm{d}}$ Fisher exact test. There were no statistically significant differences between ARGT and SSRI groups $(P>0.05)$.

reduction in plasma $\mathrm{ACTH}$ levels did not reach statistical significance, especially in GAD and OCD patients. This outcome supports findings of others on cognitive-behavioral stress management $(1,2)$, which also reported a reduction in blood cortisol concentration following psychotherapy. However, this is in contrast to a recent study from our group on patients with GAD who received Chinese Taoist Psychotherapy for 6 months, which revealed an overall improvement in anxiety symptoms and a significant decrease in ACTH levels, side by side with a significant increase in plasma cortisol levels (33). Neuroendocrine dysfunction is found in MDD, GAD and 
Table 2. Comparison of mean scores at baseline and after treatment.

\begin{tabular}{lrrr}
\hline Group & Pre & Post & $t$ \\
\hline ARGT $(n=54)$ & & & \\
HAMD scores for MDD patients $(n=19)$ & $27.95 \pm 9.62$ & $2.79 \pm 2.68^{*}$ & 18.411 \\
HAMA scores for GAD patients $(n=19)$ & $22.58 \pm 6.09$ & $3.89 \pm 2.45^{*}$ & 13.989 \\
Y-BOCS scores for OCD patients $(n=16)$ & $26.06 \pm 6.42$ & $15.94 \pm 4.73^{*}$ & 5.465 \\
SSRI $(n=55)$ & & & \\
HAMD scores for MDD patients $(n=19)$ & $28.32 \pm 4.71$ & $6.68 \pm 4.00^{*}$ & 20.092 \\
HAMA scores for GAD patients $(n=19)$ & $18.79 \pm 4.21$ & $4.36 \pm 3.48^{*}$ & 15.815 \\
Y-BOCS scores for OCD patients $(n=17)$ & $29.12 \pm 6.76$ & $20.71 \pm 5.89^{*}$ & 4.792 \\
\hline
\end{tabular}

Data are reported as means \pm SD. ARGT = attribution retraining group therapy; SSRI = selective serotonin reuptake inhibitors; MDD = major depressive disorder; GAD = generalized anxiety disorder; OCD = obsessive-compulsive disorder; HAMD = Hamilton Depression Scale; HAMA = Hamilton Anxiety Scale; Y-BOCS $=$ Yale-Brown Obsessive-Compulsive Scale. ${ }^{*} \mathrm{P}<0.001$ compared to Pre (paired-samples $t$-test).

Table 3. Comparison of plasma biological index levels in each group at baseline and after treatment.

\begin{tabular}{|c|c|c|c|}
\hline & Pre & Post & Statistics \\
\hline \multicolumn{4}{|c|}{ MDD ARGT group $(n=19)$} \\
\hline 5-HT $(\mu \mathrm{g} / \mathrm{L})$ & $87.48 \pm 60.69$ & $133.70 \pm 143.80$ & $-1.663^{a}$ \\
\hline $\mathrm{NE}(\mu \mathrm{g} / \mathrm{L})$ & $12.02 \pm 4.55$ & $12.40 \pm 5.66$ & $-0.266^{a}$ \\
\hline ACTH (ng/L) & $28.73 \pm 28.47$ & $27.36 \pm 16.48$ & $-0.040^{\mathrm{b}}$ \\
\hline Cortisol $(\mu \mathrm{g} / \mathrm{L})$ & $373.30 \pm 133.11$ & $284.30 \pm 99.49^{*}$ & $3.145^{a}$ \\
\hline \multicolumn{4}{|c|}{ MDD SSRI group $(\mathrm{n}=19)$} \\
\hline 5-HT $(\mu \mathrm{g} / \mathrm{L})$ & $85.14 \pm 28.69$ & $139.23 \pm 78.36^{*}$ & $-2.923^{a}$ \\
\hline $\mathrm{NE}(\mu \mathrm{g} / \mathrm{L})$ & $12.46 \pm 4.25$ & $15.87 \pm 6.28^{*}$ & $-2.333^{a}$ \\
\hline ACTH (ng/L) & $46.53 \pm 75.64$ & $45.39 \pm 59.99$ & $-1.231^{\mathrm{b}}$ \\
\hline Cortisol $(\mu \mathrm{g} / \mathrm{L})$ & $317.52 \pm 80.08$ & $288.09 \pm 128.19$ & $0.753^{\mathrm{a}}$ \\
\hline \multicolumn{4}{|c|}{ GAD ARGT group $(n=19)$} \\
\hline 5-HT $(\mu \mathrm{g} / \mathrm{L})$ & $106.03 \pm 55.11$ & $151.34 \pm 167.23$ & $-0.805^{b}$ \\
\hline $\mathrm{NE}(\mu \mathrm{g} / \mathrm{L})$ & $13.90 \pm 4.45$ & $12.05 \pm 4.13$ & $1.960^{\mathrm{a}}$ \\
\hline ACTH (ng/L) & $45.34 \pm 47.81$ & $33.33 \pm 32.13$ & $-1.811^{b}$ \\
\hline Cortisol ( $\mu \mathrm{g} / \mathrm{L})$ & $367.35 \pm 122.23$ & $273.93 \pm 112.69^{*}$ & $2.785^{\mathrm{a}}$ \\
\hline \multicolumn{4}{|c|}{ GAD SSRI group $(n=19)$} \\
\hline 5-HT $(\mu \mathrm{g} / \mathrm{L})$ & $91.86 \pm 60.22$ & $90.48 \pm 41.56$ & $0.100^{\mathrm{a}}$ \\
\hline $\mathrm{NE}(\mu \mathrm{g} / \mathrm{L})$ & $13.96 \pm 6.11$ & $12.14 \pm 6.06$ & $1.159^{a}$ \\
\hline ACTH (ng/L) & $29.54 \pm 18.74$ & $27.91 \pm 11.28$ & $-0.799^{b}$ \\
\hline Cortisol $(\mu \mathrm{g} / \mathrm{L})$ & $298.59 \pm 94.76$ & $286.37 \pm 67.21$ & $0.586^{\mathrm{a}}$ \\
\hline \multicolumn{4}{|c|}{ OCD ARGT group $(\mathrm{n}=16)$} \\
\hline 5-HT $(\mu \mathrm{g} / \mathrm{L})$ & $110.15 \pm 85.05$ & $92.43 \pm 55.30$ & $0.990^{\mathrm{a}}$ \\
\hline $\mathrm{NE}(\mu \mathrm{g} / \mathrm{L})$ & $11.25 \pm 4.68$ & $11.41 \pm 3.89$ & $-0.118^{a}$ \\
\hline ACTH (ng/L) & $44.76 \pm 56.58$ & $24.49 \pm 11.42$ & $-1.551^{b}$ \\
\hline Cortisol ( $\mu \mathrm{g} / \mathrm{L})$ & $367.84 \pm 117.78$ & $266.04 \pm 105.84^{*}$ & $2.877^{\mathrm{a}}$ \\
\hline \multicolumn{4}{|c|}{ OCD SSRI group $(\mathrm{n}=17)$} \\
\hline 5-HT $(\mu \mathrm{g} / \mathrm{L})$ & $99.63 \pm 68.41$ & $232.61 \pm 306.63^{*}$ & $-2.959^{b}$ \\
\hline $\mathrm{NE}(\mu \mathrm{g} / \mathrm{L})$ & $14.09 \pm 5.47$ & $12.21 \pm 5.37$ & $1.096^{\mathrm{a}}$ \\
\hline ACTH (ng/L) & $26.85 \pm 16.34$ & $27.09 \pm 8.66$ & $-0.077^{a}$ \\
\hline Cortisol $(\mu \mathrm{g} / \mathrm{L})$ & $340.78 \pm 102.91$ & $318.14 \pm 92.98$ & $0.673^{a}$ \\
\hline
\end{tabular}

Data are reported as means \pm SD. ARGT = attribution retraining group therapy; SSRI = selective serotonin reuptake inhibitors; MDD = major depressive disorder; GAD = generalized anxiety disorder; OCD = obsessive-compulsive disorder. 5 - $\mathrm{HT}=$ serotonin; $\mathrm{NE}=$ norepinephrine; ACTH $=$ adrenocorticotropic hormone. ${ }^{a}$ Paired-samples $t$-test; ' Wilcoxon test. ${ }^{*} \mathrm{P}<0.05$ compared to Pre (pairedsamples $t$-test). 
Table 4. Comparison of score reductions of plasma biological index levels between the ARGT and SSRI groups.

\begin{tabular}{|c|c|c|c|}
\hline & ARGT group & SSRI group & Statistics \\
\hline MDD group & $n=19$ & $\mathrm{n}=19$ & \\
\hline 5-HT ( $\mu \mathrm{g} / \mathrm{L})$ & $-46.22 \pm 121.17$ & $-54.09 \pm 76.30$ & $0.230^{\mathrm{a}}$ \\
\hline $\mathrm{NE}(\mu \mathrm{g} / \mathrm{L})$ & $-0.38 \pm 6.25$ & $-3.41 \pm 6.04$ & $1.478^{\mathrm{a}}$ \\
\hline ACTH (ng/L) & $1.56 \pm 25.24$ & $8.99 \pm 31.38$ & $0.326^{b}$ \\
\hline Cortisol $(\mu \mathrm{g} / \mathrm{L})$ & $89.00 \pm 123.35$ & $29.43 \pm 161.19$ & $1.253^{\mathrm{a}}$ \\
\hline GAD group & $\mathrm{n}=19$ & $\mathrm{n}=19$ & \\
\hline 5-HT ( $\mu \mathrm{g} / \mathrm{L})$ & $-42.78 \pm 181.65$ & $6.56 \pm 68.09$ & $-0.248^{b}$ \\
\hline $\mathrm{NE}(\mu \mathrm{g} / \mathrm{L})$ & $1.85 \pm 4.12$ & $2.07 \pm 7.81$ & $-1.781^{a}$ \\
\hline ACTH (ng/L) & $16.50 \pm 36.81$ & $2.13 \pm 20.55$ & $-1.617^{\mathrm{b}}$ \\
\hline Cortisol $(\mu \mathrm{g} / \mathrm{L})$ & $93.42 \pm 146.19$ & $6.08 \pm 99.80^{*}$ & $2.151^{a}$ \\
\hline OCD group & $\mathrm{n}=16$ & $\mathrm{n}=17$ & \\
\hline 5-HT $(\mu \mathrm{g} / \mathrm{L})$ & $17.72 \pm 71.60$ & $-135 \pm 249.59$ & $-1.776^{b}$ \\
\hline $\mathrm{NE}(\mu \mathrm{g} / \mathrm{L})$ & $-0.16 \pm 5.48$ & $1.87 \pm 7.04$ & $-0.922^{a}$ \\
\hline ACTH (ng/L) & $8.28 \pm 20.42$ & $-0.23 \pm 12.35$ & $1.438^{a}$ \\
\hline Cortisol $(\mu \mathrm{g} / \mathrm{L})$ & $101.80 \pm 141.54$ & $22.64 \pm 138.67^{*}$ & $-2.306^{a}$ \\
\hline
\end{tabular}

Data are reported as means \pm SD. ARGT = attribution retraining group therapy; SSRI = selective serotonin reuptake inhibitors; MDD $=$ major depressive disorder; GAD = generalized anxiety disorder; OCD = obsessive-compulsive disorder; 5 - $\mathrm{HT}=$ serotonin; $\mathrm{NE}=$ norepinephrine; ACTH $=$ adrenocorticotropic hormone. ${ }^{a}$ Independent-samples $t$-test; ${ }^{b}$ Mann-Whitney U-test. ${ }^{*} \mathrm{P}<0.05$ compared to the ARGT group (independent-samples $t$-test).

OCD patients. The different change directions between the present study and our previous one were possibly the result of different baseline cortisol and ACTH levels.

Plasma cortisol and ACTH levels may reflect HPA axis function. Some studies support HPA axis over-activation with a concurrent increase in plasma cortisol and ACTH in patients with MDD, GAD, or OCD (8). On the other hand, other studies propose a decrease in plasma cortisol concentrations, arguing that the functions of the HPA axis differ during different phases, subtypes and severity levels of the disease process $(9,32,33)$. Hence, dysfunction of the HPA axis is a more precise psychopathological description of the three diseases. Because of the possible different conditions at baseline, the effects of successful psychotherapy mentioned above on plasma cortisol concentrations may increase as well as decrease. These fluctuating changes may reflect the trend towards normalization of an abnormal HPA axis. Furthermore, the hormones of the HPA axis, such as cortisol and ACTH, are regarded as stress hormones due to their close association with stress (33). Psychotherapy is designed to adjust maladaptive attributional styles and other cognitive and behavioral responses to stressful events using various psychological skills. Current results concerning the possible role of psychotherapy in the normalization of HPA axis hormones are evidence that biological function may be improved with the improvement of psychological function by psychotherapy.

The second major finding of the present study was that there were no significant changes in cortisol or ACTH levels after SSRI between baseline and the 8th week and compared to psychotherapy. This outcome agrees with another study, which found that overactivity of the HPA axis may be one factor associated with a slower response to fluoxetine (34). The authors of the cited study hypothesized that SSRI antidepressants may not be the best therapy for MDD patients with overactivity of the HPA axis (34). On the contrary, there have also been data suggesting that antidepressants reduce cortisol levels by decreasing the sensitivity of glucocorticoid receptors (GR) (35). Hernández et al. (36) reported a significant reduction in cortisol levels in MDD patients after administration of SSRI for 52 weeks, although only a partial re-establishment of HPA axis function was achieved. It can be seen that the effect of SSRI on the HPA axis is unstable, a fact possibly due to the specific physiological targets of SSRI.

The third major finding of the present study was that plasma 5-HT levels significantly increased in MDD, GAD, and OCD patients after SSRI treatment. SSRI can block the reuptake of 5-HT by presynaptic neurons, allowing more 5$\mathrm{HT}$ to be available to the postsynaptic receptor. The patients receiving $A R G T$ in this study did not show significant changes in plasma 5-HT or NE levels, suggesting different mechanisms of action between the two systems.

There are complex functional interactions between 5$\mathrm{HT}$ and the HPA axis. Cortisol can modulate 5-HT function in the central nervous system by activating GR and the mineralocorticoid receptor (MR). Elevated 5-HT may also influence the HPA axis indirectly via the increase in cortisol self-inhibition resulting in an overall reduction of cortisol levels. Conversely, normalization of the HPA axis may influence 5-HT function indirectly by adjusting the antagonism between $\mathrm{GR}$ and $\mathrm{MR}$, and 
regulate cortisol levels further by self-inhibition as well. Therefore, treatments, which target the 5-HT system or HPA axis may result in an integrated effect on the HPA axis and 5 -HT systems. This phenomenon could account for the reduction in plasma cortisol levels observed in the SSRI group side by side with an increase in plasma $5-\mathrm{HT}$ levels in the patients treated with ARGT even though the differences did not reach statistical significance.

This study has some shortcomings. Firstly, plasma neurotransmitter concentrations may not accurately reflect the actual neurotransmitter levels in the central nervous system. Secondly, this study only evaluated changes over a short period of time. The levels of neurotransmitters in the cerebrospinal fluid may provide a more accurate measure of the absolute neurotransmitter levels but this approach is invasive and not without a significant risk to the patient. The long-term neurobiological effect of psychotherapy on MDD, GAD and OCD patients is also difficult to measure due to the dropout problem in outpatient clinics.

\section{References}

1. Cruess DG, Antoni MH, McGregor BA, Kilbourn KM, Boyers $\mathrm{AE}$, Alferi SM, et al. Cognitive-behavioral stress management reduces serum cortisol by enhancing benefit finding among women being treated for early stage breast cancer. Psychosom Med 2000; 62: 304-308.

2. Antoni MH. Stress management effects on psychological, endocrinological, and immune functioning in men with HIV infection: empirical support for a psychoneuroimmunological model. Stress 2003; 6: 173-188, doi: 10.1080/ 1025389031000156727.

3. Jeong YJ, Hong SC, Lee MS, Park MC, Kim YK, Suh CM. Dance movement therapy improves emotional responses and modulates neurohormones in adolescents with mild depression. Int J Neurosci 2005; 115: 1711-1720, doi: $10.1080 / 00207450590958574$.

4. Lucki I. The spectrum of behaviors influenced by serotonin. Biol Psychiatry 1998; 44: 151-162, doi: 10.1016/S00063223(98)00139-5.

5. Connor KM, Davidson JR. Generalized anxiety disorder: neurobiological and pharmacotherapeutic perspectives. Biol Psychiatry 1998; 44: 1286-1294, doi: 10.1016/S00063223(98)00285-6.

6. Gold PW, Chrousos GP. Organization of the stress system and its dysregulation in melancholic and atypical depression: high versus low CRH/NE states. Mol Psychiatry 2002; 7: 254-275, doi: 10.1038/sj.mp.4001032.

7. Arborelius L, Owens MJ, Plotsky PM, Nemeroff CB. The role of corticotropin-releasing factor in depression and anxiety disorders. J Endocrinol 1999; 160: 1-12, doi: 10.1677/joe.0.1600001.

8. Kluge M, Schussler P, Kunzel HE, Dresler M, Yassouridis A, Steiger A. Increased nocturnal secretion of ACTH and cortisol in obsessive compulsive disorder. J Psychiatr Res 2007; 41: 928-933, doi: 10.1016/j.jpsychires.2006.08.005.

9. Kaestner F, Hettich M, Peters M, Sibrowski W, Hetzel G, Ponath G, et al. Different activation patterns of proinflam-
On the whole, this study demonstrated that ARGT and SSRI can both improve symptoms in MDD, GAD, and OCD patients. Plasma cortisol levels dropped significantly in patients with MDD, GAD, and OCD patients after ARGT. Plasma 5-HT levels increased significantly in MDD, GAD, and OCD patients after SSRI treatment. This finding suggests that ARGT may modulate plasma cortisol levels and affect the HPA axis, as opposed to SSRIs, which may up-regulate plasma $5-\mathrm{HT}$ levels via a different pathway to produce an overall improvement in the clinical condition of the patients.

\section{Acknowledgments}

Research supported by the National Science and Technology Support Projects, China (\#2009BA177B07), the National Natural Science Foundation for Youth of China (\#81201064), and the Nanjing Medical Science and Technique Development Youth Foundation, China (\#QYK09184).

matory cytokines in melancholic and non-melancholic major depression are associated with HPA axis activity. J Affect Disord 2005; 87: 305-311, doi: 10.1016/j.jad.2005.03.012.

10. Parker KJ, Schatzberg AF, Lyons DM. Neuroendocrine aspects of hypercortisolism in major depression. Horm Behav 2003; 43: 60-66, doi: 10.1016/S0018-506X(02)00016-8.

11. Schulz $\mathrm{P}$, Kaspar $\mathrm{CH}$. Neuroendocrine and psychological effects of restricted environmental stimulation technique in a flotation tank. Biol Psychol 1994; 37: 161-175, doi: 10.1016/ 0301-0511(94)90029-9.

12. Garvey MJ, Hollon SD, Evans MD. Examination of preand posttreatment MHPG during cognitive therapy. Neuropsychobiology 1992; 26: 182-185, doi: 10.1159/ 000118917.

13. Zhang YL, Zhao JP, Yang DS, Lu XH, Zhang XH, Cao YP. A longitudinal study of monoamine neurotransmitter, neuroendocrine and immune parameter in the patients with generalized anxiety disorder [in Chinese]. Chinese J Psychiatry 2000; 33: 200-202.

14. Abelson JL, Liberzon I, Young EA, Khan S. Cognitive modulation of the endocrine stress response to a pharmacological challenge in normal and panic disorder subjects. Arch Gen Psychiatry 2005; 62: 668-675, doi: 10.1001/ archpsyc.62.6.668.

15. Forsterling F. Attributional retraining: a review. Psychol Bull 1985; 98: 495-512, doi: 10.1037/0033-2909.98.3.495.

16. Hilt L. Attribution retraining for therapeutic change: theory, practice, and future directions. Imagination, Cognition and Personality 2004; 23: 289-307, doi: 10.2190/WC91-VXQKN665-2J2Y.

17. Abramson LY, Seligman ME, Teasdale JD. Learned helplessness in humans: critique and reformulation. $J$ Abnorm Psychol 1978; 87: 49-74, doi: 10.1037/0021-843X.87.1.49.

18. Seligman ME, Metalsky G, Alloy LB. Hopelessness depression: a theory-based subtype of depression. Psychological 
Rev 1989; 96: 358-372, doi: 10.1037/0033-295X.96.2.358.

19. Metalsky GI, Joiner TE Jr, Hardin TS, Abramson LY. Depressive reactions to failure in a naturalistic setting: a test of the hopelessness and self-esteem theories of depression. J Abnorm Psychol 1993; 102: 101-109, doi: 10.1037/ 0021-843X.102.1.101.

20. Haugen R, Lund T. Self-concept, attributional style and depression. Educational Psychol 2002; 22: 305-315, doi: 10.1080/01443410220138539.

21. Waikar SV, Craske MG. Cognitive correlates of anxious and depressive symptomatology: an examination of the Helplessness/Hopelessness model. J Anxiety Disord 1997; 11: 1-16, doi: 10.1016/S0887-6185(96)00031-X.

22. Luten AG, Ralph JA, Mineka S. Pessimistic attributional style: is it specific to depression versus anxiety versus negative affect? Behav Res Ther 1997; 35: 703-719, doi: 10.1016/S0005-7967(97)00027-2.

23. Ahrens AH, Haaga DAF. The specificity of attributional style and expectations to positive and negative affectivity, depression, and anxiety. Cognitive Ther Res 1993; 17: 8398, doi: 10.1007/BF01172742.

24. Wang C, Zhang N. Get back the peace of mind. In: Wang W (Editor), Case analysis: counselling and psychotherapy. Beijing: People's Medical Publishing House; 2008. p 227-250.

25. Zhang N, Wang C. Group attributive training in undergraduates with high self-evaluating depressive score [in Chinese]. J Clin Psychiatry 2007; 17: 229-231.

26. Zhang J, Wang C, Zhang N, Wu X, Guo SW, Ou HX. Curative effects of the attribution retraining on major depression disorder, anxiety disorder and obsessive-compulsive disorder [in Chinese]. J Clin Psychiatry 2010; 6: 371-373.

27. Wang C, Zhang J, Zhang N, Zhang YL. Effectiveness and comparison of attribution retraining group therapy: a clinical trial of outpatients with major depression disorder, generalized anxiety disorder, and obsessive-compulsive disorder. $J$ Biomed Res 2011; 25: 348-355, doi: 10.1016/S16748301(11)60046-8.
28. First MB, Spitzer RL, Gibbon M, Williams JBW. Structured clinical interview for DSM-IV axis I disorders, patient edition. New York: Biometrics Research Department, New York Psychiatric Institute; 1994.

29. Hamilton M. A rating scale for depression. J Neurol Neurosurg Psychiatry 1960; 23: 56-62, doi: 10.1136/ jnnp.23.1.56.

30. Hamilton $\mathrm{M}$. The assessment of anxiety states by rating. $\mathrm{Br}$ J Med Psychol 1959; 32: 50-55, doi: 10.1111/j.20448341.1959.tb00467.x.

31. Goodman WK, Price LH, Rasmussen SA, Mazure C, Fleischmann RL, Hill CL, et al. The Yale-Brown Obsessive Compulsive Scale. I. Development, use, and reliability. Arch Gen Psychiatry 1989; 46: 1006-1011, doi: 10.1001/archpsyc.1989.01810110048007.

32. Nutt DJ, Forshall S, Bell C, Rich A, Sandford J, Nash J, et al. Mechanisms of action of selective serotonin reuptake inhibitors in the treatment of psychiatric disorders. Eur Neuropsychopharmacol 1999; 9 (Suppl 3): S81-S86, doi: 10.1016/S0924-977X(99)00030-9.

33. Zhang N, Hou G, Fang Q. Effects of stress on episode of depression and changes of hormones [in Chinese]. Chinese Mental Health J 2003; 17: 610-612.

34. Young EA, Altemus M, Lopez JF, Kocsis JH, Schatzberg $A F$, DeBattista $C$, et al. HPA axis activation in major depression and response to fluoxetine: a pilot study. Psychoneuroendocrinology 2004; 29: 1198-1204, doi: 10.1016/j.psyneuen.2004.02.002.

35. Okuyama-Tamura M, Mikuni M, Kojima I. Modulation of the human glucocorticoid receptor function by antidepressive compounds. Neurosci Lett 2003; 342: 206-210, doi: 10.1016/S0304-3940(03)00261-1.

36. Hernández ME, Mendieta D, Martinez-Fong D, Loria F, Moreno J, Estrada I, et al. Variations in circulating cytokine levels during 52 week course of treatment with SSRI for major depressive disorder. Eur Neuropsychopharmacol 2008; 18: 917-924, doi: 10.1016/j.euroneuro.2008.08.001. 